OPEN ACCESS

Edited by:

Efrat Neter

Ruppin Academic Center, Israel

Reviewed by:

Michele Okun

University of Colorado Colorado

Springs, United States

Dena Sadeghi Bahmani,

University of Alabama at Birmingham,

United States

*Correspondence:

Alexandra Main

amain@ucmerced.edu

Specialty section:

This article was submitted to

Health Psychology,

a section of the journa

Frontiers in Psychology

Received: 06 January 2021 Accepted: 29 April 2021

Published: 21 May 2021

Citation:

Yung ST, Main A, Walle EA, Scott RM and Chen Y (2021)

Associations Between Sleep and Mental Health Among Latina Adolescent Mothers: The Role of Social Support.

Front. Psychol. 12:647544. doi: 10.3389/fpsyg.2021.647544

\section{Associations Between Sleep and Mental Health Among Latina Adolescent Mothers: The Role of Social Support}

\author{
Shun Ting Yung, Alexandra Main*, Eric A. Walle, Rose M. Scott and Yaoyu Chen \\ Department of Psychological Sciences, University of California, Merced, Merced, CA, United States
}

Adolescent mothers experience poorer sleep than adult mothers, and Latina adolescent mothers are at greater risk of postpartum depression compared with other racial/ethnic groups. However, social support may be protective against the negative effects of poor sleep in this population. The current study examined (1) associations between the quality and quantity of Latina adolescent mothers' sleep and mental health (depressive symptoms and anxiety), and (2) whether social support buffered the effects of poor sleep on mental health. A sample of Latina adolescent mothers $(N=84)$ from an agricultural region in the United States reported on their sleep duration/quality, social support from family, friends, and significant others, and their depressive and anxiety symptoms. Results showed that adolescent mothers reported poorer sleep than pediatric recommendations, and poorer sleep quality was associated with greater depressive and anxiety symptoms. Interestingly, when adolescent mothers reported better sleep, they had fewer depressive symptoms in the context of high support from friends compared with low support from friends. Sleep is important for mental health in Latina adolescent mothers, and better sleep combined with strong social support has positive associations with mental health in this population. Findings hold implications for improving mental health in adolescent mothers.

\section{Keywords: Latinx, adolescent mothers, sleep, social support, mental health}

Adolescent mothers face unique challenges in their transition to motherhood, including higher levels of depression compared with adult mothers (Lanzi et al., 2009). Adolescent mothers are more likely than adult mothers to live in poverty, particularly in agricultural regions of the United States such as the San Joaquin Valley in California where the adolescent birthrate is nearly twice the overall rate statewide (California Department of Public Health, 2017). Though adolescent pregnancy rates are generally declining, adolescents of color experience greater health disparities and are at higher risk for unplanned pregnancies (Office of Disease Prevention and Health Promotion, n.d.), particularly Latina adolescents (Jahromi et al., 2012). Thus, it is crucial to understand factors that (a) are associated with Latina adolescent mothers' mental health, and (b) may buffer Latina adolescent mothers from poor mental health outcomes. 


\section{CHALLENGES OF ADOLESCENT MOTHERHOOD}

Adolescent mothers are more likely to experience psychological and behavioral adjustment problems than adult mothers (Chang and Fine, 2007). The need to integrate their maternal role with their individual identity development can create role conflict, confusion, and emotional distress (Birkeland et al., 2005). Over half of adolescent mothers experienced moderate to severe depressive symptoms during the first 4 years postpartum (Schmidt et al., 2006). Compared to European American mothers, Latina adolescent mothers are more vulnerable to depression (Howell et al., 2005). Sociocultural stressors, including discrimination and acculturative stress experienced during the perinatal period, may contribute to poor mental health in this population (e.g., Luis Sanchez et al., 2020). Furthermore, Latina mothers living in rural communities have a $40 \%$ greater chance of developing postpartum depression compared to White mothers (Ceballos et al., 2016). However, risk and protective factors in the context of Latina adolescent mothers' mental health remain understudied.

\section{POSTPARTUM SLEEP AND MENTAL HEALTH}

Sleep is intricately linked with physical, cognitive, and psychological health and wellbeing (e.g., Sadeghi Bahmani et al., 2018; Zhang et al., 2018). New mothers are often vulnerable to the negative effects of sleep disruptions during the postpartum period (Richter et al., 2019). Insufficient sleep is related to greater psychological distress and vulnerability to mood disorders, including anxiety, and depression (e.g., Okun et al., 2018). Researchers have found that depressive symptoms at 1-month postpartum predict worse sleep quality at 6 months, which predicts greater depressive symptoms at 6 and 12 months postpartum (Stremler et al., 2019). Disrupted sleep among postpartum mothers can develop into a "vicious cycle" of poor sleep quality and depression.

The National Sleep Foundation in the United States recommends $8-10 \mathrm{~h}$ of sleep per night for adolescents ages 1417 (Hirshkowitz et al., 2015). Adolescent mothers may be at particular risk for poor sleep because adolescents with insomnia have greater difficulty with affective regulation (Brand et al., 2016), which has implications for poor mental health, including depression and anxiety (Zhang et al., 2017). Conversely, longer sleep duration is associated with better subjective psychological well-being among adolescents (Kalak et al., 2014). Adolescents also undergo critical biological changes in sleep patterns, including sleep phase delay, a natural process in which adolescents have a tendency to go to sleep and wake later (Shochat et al., 2014). In addition to biological changes, adolescence coincides with changing environmental factors (e.g., earlier school start times, growing academic workload, less parental influence on bedtime, increased technology usage), which may contribute to decreased sleep, and in turn increase anxiety and depression (Shochat et al., 2014). Taken together, adolescent mothers may experience more mental health problems caused by sleep disruptions than adult mothers due to these normative changes. Despite these risks, the impact of sleep on adolescent mothers' mental health has not been examined to our knowledge.

\section{THE ROLE OF SOCIAL SUPPORT}

Social support from close others is an important protective factor against postpartum depression (Hahn-Holbrook et al., 2018), and may be particularly important for adolescent mothers' mental health (Oberlander et al., 2007). Previous studies have shown that social support predicts less depression among adolescent mothers (see Reid and Meadows-Oliver, 2007). Social support may be especially important for Latina adolescent mothers, as family support is central to Latinx culture (e.g., Bravo et al., 2016). For example, Latina mothers are more likely to seek support and share childcare responsibilities with family members compared to other ethnic groups (Cauce et al., 2002). Furthermore, high-quality family interactions are related to healthy sleep among Latinx adolescents (Sasser et al., 2020). Indeed, Latina adolescent mothers who live with family members experience lower parenting stress compared to their African American peers (Huang et al., 2019).

While family support may protect against negative outcomes for adolescent mothers, peers may also be an important source of support. For example, a case study showed that an adolescent mother reported greater conflict with parents and believed friends were more helpful than parents (Stiles, 2010). Moreover, the involvement of the child's father or the mother's significant other is an important source of support for adolescent mothers (Smith et al., 2013). Latina adolescent mothers report less postpartum depression when fathers are more involved with childcare (Fagan and Lee, 2010). Taken together, support from parents, friends, and significant others may all be important protective factors for adolescent mothers. However, to our knowledge, prior research has not investigated how social support may buffer the effects of poor sleep on mental health postpartum.

\section{THE PRESENT STUDY}

The present study examined sleep, mental health, and social support in a sample of Latina adolescent mothers from an agricultural, predominantly rural region of the United States. The aims of the present study were to examine: (1) the quality and quantity of Latina adolescent mothers' sleep, (2) associations between sleep and mental health (depressive symptoms and anxiety), and (3) whether social support from family, friends, and significant others buffered the effects of sleep duration/quality on mental health. We hypothesized that adolescent mothers would have lower sleep quality and quantity compared with pediatric recommendations, and that lower quality/quantity of sleep would be associated with higher depressive symptoms and anxiety. We also hypothesized that social support from family, friends, and significant others would buffer the effects of sleep duration/quality on mental health among adolescent mothers. Given the unique demographics of the sample, we also explored associations between demographic characteristics 
(primary caretaker education and generation status) and sleep, mental health, and social support.

\section{MATERIALS AND METHODS}

\section{Participants}

Participants were 84 adolescent mothers, $M_{\text {age }}(S D)=17.81(0.94)$ years, who self-identified as Latina. Adolescents were eligible to participate if they were between 13 and 19 years of age, were a biological parent $(N=73,45 \%$ of children $=$ female $)$ or pregnant $(N=11)$ at the time of participation and could read or speak English or Spanish. Participants were recruited from high schools and afterschool programs in California's San Joaquin Valley. The majority of participants were born in the United States (81\%), followed by Mexico (18\%), or another country (1\%). Participants reported that their mother was born in the United States (47\%), Mexico (52\%), or another country (1\%), and that their father was born in the United States (38\%), Mexico (59\%), or another country (3\%). Over half of the adolescent mothers were single (63\%) at the time of participation, 33\% were living with their spouse or romantic partner, and $1 \%$ were married. The median education level of the adolescent's primary caretaker was a high school diploma or equivalent (see Table $\mathbf{1}$ for more descriptive information about the sample).

\section{Procedure}

The study was approved by the Institutional Review Board at the academic institution where the research was conducted. Parents provided informed consent for adolescents less than 18 years of age and adolescents provided assent (if under 18) or consent (if 18 or older). Participants completed either an online survey or a paper survey that was administered by trained researchers in a classroom setting. Questionnaires not previously used in research

TABLE 1 | Descriptive statistics of demographic, sleep, social support, and mental health variables.

\begin{tabular}{lrrrr}
\hline Variable & Min & Max & Mean & SD \\
\hline Adolescent age & 14.88 & 19.88 & 17.81 & 0.94 \\
Child age (months) & 0.79 & 48.20 & 13.42 & 9.87 \\
Primary caretaker education $^{\mathrm{a}}$ & 1.00 & 4.00 & 1.53 & 0.79 \\
Generation status $^{\text {b }}$ & 0.00 & 2.00 & 1.10 & 0.74 \\
Sleep quantity (hours) & 2.00 & 10.00 & 6.55 & 1.80 \\
Sleep quality & 1.00 & 5.00 & 3.05 & 1.07 \\
Depressive symptoms & 0.00 & 26.00 & 11.57 & 6.09 \\
Anxiety symptoms & 20.00 & 58.00 & 35.55 & 8.65 \\
Family support & 1.00 & 7.00 & 4.87 & 1.69 \\
Friend support & 1.00 & 7.00 & 4.13 & 2.18 \\
Significant other support & 1.00 & 7.00 & 5.24 & 1.70 \\
\hline
\end{tabular}

Min, minimum; Max, maximum, SD, standard deviation.

a Primary caretaker education is coded as: 1 = some high school or less, 2 = high school graduate or equivalent, 3 = some college, 4 = associates/vocational degree, 5 = bachelors degree, 6 = masters degree, $7=\mathrm{MD} / \mathrm{PhD} / \mathrm{JD}$.

${ }^{b}$ Generation status is coded as: 0 = adolescent born outside the United States,

$1=$ at least one parent born outside the United States, and 2 = both parents born in the United States. with Spanish-speaking samples were forward and back-translated by bilingual research assistants.

\section{Measures}

\section{Sleep Duration/Quality}

Questions derived from the Pittsburgh Sleep Quality Index (PSQI; Buysse et al., 1989) were used to access adolescents' sleep quality and duration. This measure has good test-re-test reliability and diagnostic sensitivity with adults and adolescents (Buysse et al., 1989; Raniti et al., 2018). Participants were asked to report on the quantity and quality of sleep in the past month by responding to the following questions: (1) "On average, how many hours of sleep do you get per night?" ( $1=$ less than $4 \mathrm{~h}$ to $5=$ more than $9 \mathrm{~h}$ ), and (2) "How would you describe the quality of your sleep on an average night?" ( 1 = very poor to $5=$ very good $)$. The items were highly correlated $(r=0.62$, $p<0.001)$; thus, a composite was created by standardizing the items and computing the mean. This variable was used in all analyses of sleep.

\section{Depressive Symptoms}

The 11-item version of the Center for Epidemiologic Studies Depression Scale (CES-D; Radloff, 1977) assessed adolescent depressive symptoms in the past week. The CES-D is a commonly used self-report measure of depressive symptoms that has been shown to have high internal consistency and validity in adolescent samples (e.g., Radloff, 1977; Stockings et al., 2015), including Latinx adolescents (Suarez-Colorado et al., 2020). Each item was rated on a scale of 0 (rarely or none of the time) to 3 (most or all the time). Sample items include, "I did not feel like eating; my appetite was poor" and "I felt everything I did was an effort." Scores were summed to create an overall depressive symptoms score, with a higher total score indicating more depressive symptoms. This scale demonstrated adequate internal consistency $(\alpha=0.75)$.

\section{Anxiety}

The 20-item State-Trait Anxiety Inventory for Children (STAIC; Spielberger et al., 1973) assessed adolescent anxiety in the past month. The STAI-C is a widely used tool to evaluate the presence and severity of current anxiety symptoms. The STAI-C has good internal consistency and high convergent and discriminative validity (Bakker et al., 1989; Beesdo et al., 2009) and is a valid measurement of anxiety in the postpartum period (Dennis et al., 2013). Each item was rated on a scale of 1 (hardly ever) to 3 (often). Sample items include, "It is difficult for me to face my problems" and "I am secretly afraid." Scores were summed to create an overall anxiety score, with a higher total score indicating more anxiety. The internal consistency coefficient was $\alpha=0.90$.

\section{Social Support}

The 12-item Multidimensional Scale of Perceived Social Support (Zimet et al., 1988) assessed social support from family, friends, and significant others. Each item was rated on a scale of 1 (very strongly disagree) to 7 (very strongly agree). Sample items include, "There is a special person who is around when I am in need" and "There is a special person in my life who cares about my feelings." This measure has been shown to be a suitable measure 
of perceived social support in Latinx adolescents (Trejos-Herrera et al., 2018). Scores were summed separately for the three sources of social support in the current study ( $\alpha=0.89,0.95$, and 0.92 for family, friends, and significant other, respectively).

\section{RESULTS}

Descriptive statistics for demographic and study variables are presented in Table 1. Variables were screened for normality; all fell within the normal range of 2 for skewness and 7 for kurtosis. Participants reported an average of $6.5 \mathrm{~h}$ of sleep per night $(S D=1.80 \mathrm{~h})$, with nearly $82 \%$ of the sample reporting fewer than $8 \mathrm{~h}$ of sleep per night. This is lower than the recommended $8-10 \mathrm{~h}$ and average $9 \mathrm{~h}$ of sleep for adolescents (Hirshkowitz et al., 2015). Moreover, nearly one third of the sample reported very poor or somewhat poor quality of sleep.

Zero-order correlations were conducted to examine relations among the demographic and study variables (see Table 2). There were no significant associations between demographic variables and sleep, mental health, or social support; thus, these variables were not included in subsequent analyses. Adolescents who reported poorer sleep reported higher depressive and anxiety symptoms. Additionally, adolescents who reported receiving more family support indicated receiving more support from friends and their significant others. There were no significant associations between social support and sleep or mental health.

To test whether social support buffered the effects of poor sleep on depressive and anxiety symptoms, 6 hierarchical multiple regression analyses were conducted with social support from each source (family, friends, and significant other) included in the models along with the interaction with sleep duration/quality. The first step of each model included the main effects of sleep and social support. The second step included the main effects and the interaction term of sleep and social support. Interaction terms were created by centering both variables around their mean and multiplying them together.

Results revealed a main effect of sleep for all models, with better sleep associated with fewer depressive and anxiety symptoms (see Table 3 ). Results also revealed a significant
TABLE 3 | Standardized hierarchical multiple regressions predicting depressive and anxiety symptoms from social support and sleep duration/quality.

\begin{tabular}{|c|c|c|c|c|}
\hline \multirow[b]{2}{*}{ Variable } & \multicolumn{2}{|c|}{ Depressive symptoms } & \multicolumn{2}{|c|}{ Anxiety symptoms } \\
\hline & $\beta$ & $\Delta R^{2}$ & $\beta$ & $\Delta R^{2}$ \\
\hline Step 1 & & 0.10 & & 0.19 \\
\hline Family support & -0.02 & & 0.05 & \\
\hline Sleep & $-0.34^{\star}$ & & $-0.47^{\star}$ & \\
\hline Step 2 & & 0.09 & & 0.19 \\
\hline Family support $\times$ Sleep & -0.02 & & 0.08 & \\
\hline Step 1 & & 0.10 & & 0.21 \\
\hline Friend support & -0.07 & & 0.04 & \\
\hline Sleep & $-0.33^{\star}$ & & $-0.46^{\star}$ & \\
\hline Step 2 & & 0.14 & & 0.21 \\
\hline Friend support X Sleep & $-0.22^{\star}$ & & -0.07 & \\
\hline Step 1 & & 0.12 & & 0.19 \\
\hline Significant other support & -0.15 & & -0.02 & \\
\hline Sleep duration/quality & $-0.34^{\star}$ & & $-0.45^{\star}$ & \\
\hline Step 2 & & 0.11 & & 0.18 \\
\hline $\begin{aligned} & \text { Significant other support } \\
\times & \text { Sleep }\end{aligned}$ & 0.05 & & -0.02 & \\
\hline
\end{tabular}

interaction between sleep and social support from friends predicting depressive symptoms $(B=-0.67, p=0.03)$. Plotting of the interaction using simple slopes analysis showed that adolescents reported fewer depressive symptoms when sleep duration/quality was high compared with poorer sleep, but only if support from friends was also high (see Figure 1). No other significant interactions between social support and sleep predicting mental health were present.

\section{DISCUSSION}

The current study fills an important gap in the literature by examining relations between sleep duration and quality, social support (family, friends, and significant other) and mental health (depressive and anxiety symptoms) in Latina adolescent mothers in a predominantly rural, agricultural region of the United States.

TABLE 2 | Correlations between demographic variables, sleep, social support, and mental health variables.

\begin{tabular}{|c|c|c|c|c|c|c|c|c|c|c|}
\hline Variable & 1 & 2 & 3 & 4 & 5 & 6 & 7 & 8 & 9 & 10 \\
\hline 1. Adolescent age & - & & & & & & & & & \\
\hline 2. Child age (months) & $0.32^{\star \star}$ & - & & & & & & & & \\
\hline 3. Child gender ( $1=$ female, $2=$ male $)$ & -0.03 & 0.06 & - & & & & & & & \\
\hline 4. Primary caretaker education & 0.08 & -0.19 & -0.05 & - & & & & & & \\
\hline 5. Generation status & 0.06 & -0.13 & 0.01 & 0.18 & - & & & & & \\
\hline 6. Adolescent sleep & -0.13 & -0.09 & 0.04 & 0.00 & -0.13 & - & & & & \\
\hline 7. Depressive symptoms & 0.08 & 0.09 & -0.02 & -0.11 & -0.15 & $-0.35^{\star \star}$ & - & & & \\
\hline 8. Anxiety symptoms & 0.02 & -0.04 & -0.07 & 0.23 & 0.13 & $-0.46^{\star \star}$ & $0.57^{\star \star}$ & - & & \\
\hline 9. Family support & 0.06 & -0.14 & -0.08 & 0.05 & -0.04 & 0.21 & -0.08 & -0.05 & - & \\
\hline 10. Friend support & 0.04 & -0.10 & 0.01 & -0.07 & 0.04 & 0.17 & -0.12 & -0.04 & $0.49^{\star \star}$ & - \\
\hline 11. Significant other support & 0.07 & -0.12 & -0.10 & 0.09 & -0.27 & 0.03 & -0.16 & -0.03 & $0.61^{\star \star}$ & $0.54^{\star \star}$ \\
\hline
\end{tabular}

${ }^{* *} p<0.01,{ }^{*} p<0.05$. 


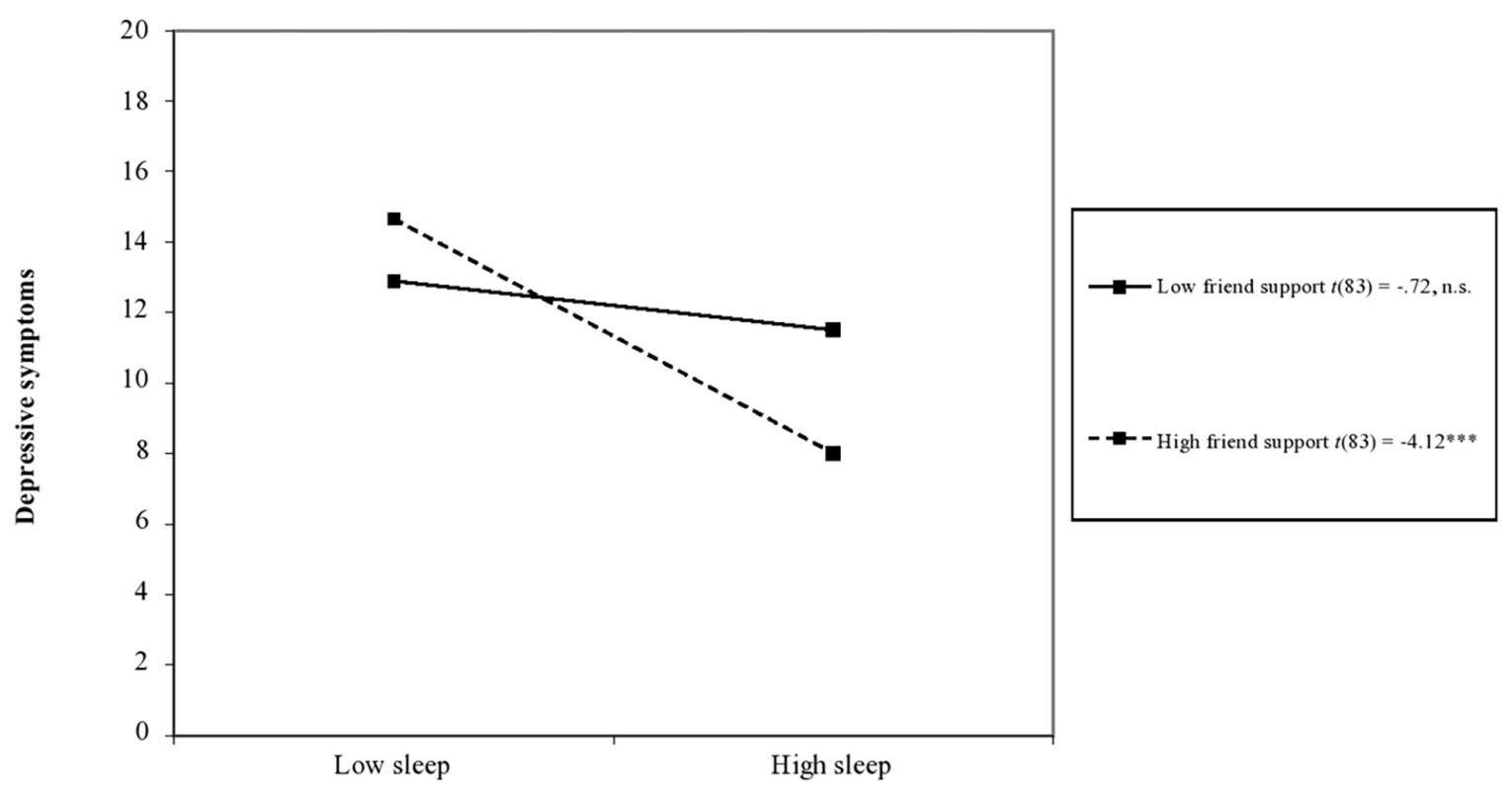

FIGURE 1 | Interaction between sleep duration/quality and social support from friends predicting adolescent parents' depressive symptoms. Notes: $t$-values are unstandardized simple slopes. n.s., not significant, ${ }^{* * *} p<0.001$.

Findings demonstrated that fewer hours of sleep and lower quality of sleep were linked with higher depressive symptoms and anxiety, and that social support from friends buffered the association between poor sleep and depressive symptoms. These findings are discussed in detail below.

\section{Sleep, Mental Health, and Social Support}

In line with previous studies, adolescent mothers in the current sample were at risk for inadequate sleep. The average number of hours of sleep in the past month for the sample in the current study is lower than pediatric recommendations $(8-10 \mathrm{~h}$ of sleep per night; Hirshkowitz et al., 2015), and considerably lower than the average number of hours of sleep reported in a large study of Canadian adolescents, which found that approximately one-third of adolescents reported insufficient sleep (Chaput and Janssen, 2016). It is common for new mothers to report insomnia, sleep deficiency or sleep disturbances (e.g., Reichner, 2015). This may be particularly true for adolescent mothers because adolescence is a critical developmental period when sleep patterns undergo significant changes (Shochat et al., 2014).

Consistent with hypotheses, adolescent mothers who reported worse sleep (fewer hours and lower quality) reported higher depressive symptoms and anxiety. This is consistent with previous studies with adult mothers (e.g., Figueiredo et al., 2007) and findings that inadequate sleep is associated with depression and anxiety in adolescence (e.g., Shochat et al., 2014). The current study extends these findings to a sample of Latina adolescent mothers, who are at particular risk of mental health problems in the postpartum period (Bámaca-Colbert et al., 2011).

We found partial support for our second hypothesis that social support would buffer the effects of poor sleep on adolescent mothers' mental health, but only for social support from peers. Specifically, adolescents reported fewer depressive symptoms in the context of both better sleep and higher social support from friends. Growing reliance on peers rather than parents for social support is an important transition in adolescence (Brown and Larson, 2009), particularly when conflict with parents is high (Stiles, 2010). However, social support from family and significant others did not buffer the effects of poor sleep on mental health. These findings underscore the importance of considering multiple biological and environmental factors that undergo change during adolescence.

Somewhat surprisingly, primary caretaker education and generation status (i.e., whether adolescent mothers were born in the United States) were not associated with participants' quality of sleep, mental health or social support. Low socioeconomic status has been identified as a mental health risk factor for first-time mothers (Goyal et al., 2010), and prior research has shown that U.S.-born Latinx youth and those with U.S.-born parents are more likely than their non-U.S.-born counterparts to engage in risky behaviors, including tobacco, drug, and alcohol use and have earlier age of sexual initiation (e.g., Kaplan et al., 2002). Future research could explore other cultural factors, such as cultural values (e.g., familismo) and acculturative processes, to test whether maintenance of traditional cultural values or acculturation are linked with social support and may buffer Latina adolescent mothers from poor mental health outcomes.

\section{Limitations, Future Directions for Research, and Clinical Implications}

The current study is an important first step in understanding the role of sleep in Latina adolescent mothers' mental health. However, some limitations warrant mentioning. First, the current study relied on self-report and assessed study variables retrospectively, which may be subject to bias. Future research 
could use more objective measures of sleep, including sleep tracking devices and observed measures of relationship quality between adolescent mothers and social support figures. Second, the cross-sectional design precludes examining developmental changes in associations between adolescent mothers' sleep quality and mental health. Finally, though the current study sheds light on associations between sleep, social support, and mental health within an understudied population (i.e., Latina adolescent mothers), comparisons to other groups (e.g., other ethnicities, urban samples) would help identify potentially unique protective factors within this population.

Our findings have important implications for future research directions. It is important to stress the multifaceted impact of affective disorders associated with reduced sleep quality. For example, postpartum depression has been found to have negative consequences for the quality of mother-child interactions (Slomian et al., 2019). These consequences include poor physical and psychological health, increased risk behaviors, and worsened interpersonal relationships for mothers. Moreover, maternal postpartum depression also has negative consequences for a host of infant outcomes due to reduced caregiving sensitivity, including physical, behavioral, cognitive, linguistic, and socioemotional development (e.g., Grace et al., 2003; Lanzi et al., 2009; Slomian et al., 2019). The broad-reaching impact of adolescent mothers' mental health on their children and social networks is an important topic for future investigation.

Finally, this study holds implications for understanding sleep and mental health in Latina adolescent mothers. Healthcare practitioners should be aware of the importance of adequate sleep for adolescent mothers and assess postpartum sleep duration and quality. Our findings also indicate the importance of peer support that adolescent mothers receive. Interventions focused

\section{REFERENCES}

Bakker, F. C., Van Wieringen, P. C. W., Van der Ploeg, H. M., and Spielberger, C. D. (1989). Manual for the Dutch State-Trait Anxiety Inventory for Children. Lisse: Swets en Zeitlinger BV.

Bámaca-Colbert, M. Y., Gayles, J. G., and Lara, R. (2011). Family correlates of adjustment profiles in Mexican- origin female adolescents. Hisp. J. Behav. Sci. 33, 123-151. doi: 10.1177/0739986311403724

Beesdo, K., Knappe, S., and Pine, D. S. (2009). Anxiety and anxiety disorders in children and adolescents: developmental issues and implications for DSM-V. Psychiatr. Clin. North Am. 32, 483-524. doi: 10.1016/j.psc.2009. 06.002

Birkeland, R., Thompson, J. K., and Phares, V. (2005). Adolescent motherhood and postpartum depression. J. Clin. Child Adoles. Psychol. 34, 292-300. doi: 10.1207/s15374424jccp3402_8

Brand, S., Kirov, R., Kalak, N., Gerber, M., Schmidt, N. B., Lemola, S., et al. (2016). Poor sleep is related to lower emotional competence among adolescents. Behav. Sleep Med. 14, 602-614. doi: 10.1080/15402002.2015.1048450

Bravo, A. J., Kelley, M. L., and Hollis, B. F. (2016). Social Support, depressive symptoms, and hazardous alcohol use among navy members: an examination of social support as a protective factor across deployment. J. Soc. Clin. Psychol. 35, 693-704. doi: 10.1521/jscp.2016.35.8.693

Brown, B. B., and Larson, J. (2009). "Peer relationships in adolescence," in Handbook of Adolescent Psychology, 3rd Edn, Vol. 2, eds R. M. Lerner and L. Steinberg (Hoboken, NJ: Wiley), 74-103.

Buysse, D. J., Reynolds, C. F. III, Monk, T. H., Berman, S. R., and Kupfer, D. J. (1989). The pittsburgh sleep quality index: a new instrument for psychiatric practice and research. Psychiatry Res. 28, 193-213. doi: 10.1016/0165-1781(89) 90047-4 on reducing psychological distress in adolescent mothers should consider ways to strengthen their support from friends and peer network to promote positive mental health in this atrisk population.

\section{DATA AVAILABILITY STATEMENT}

The raw data supporting the conclusions of this article will be made available by the authors, without undue reservation.

\section{ETHICS STATEMENT}

The studies involving human participants were reviewed and approved by The UC Merced Institutional Review Board, holding Department of Health and Human Services Federalwide Assurance \#00005105, has reviewed and approved this study. It was approved by Ramesh Balasubramaniam, the Chair of the IRB. Written informed consent to participate in this study was provided by the participants' legal guardian/next of kin. Participants who were 18 years old or above provided informed consent themselves.

\section{AUTHOR CONTRIBUTIONS}

SY, AM, EW, and RS performed the material preparation and data collection and analysis. SY wrote the first draft of the manuscript. YC contributed to the literature review. All authors commented on previous versions of the manuscript, read and approved the final manuscript.

California Department of Public Health (2017). Births: 2013-2015, Birth Statistical Master File. Trenton, NJ: Center for Health Statistics and Informatics.

Cauce, A. M., Domenech-Rodríguez, M., Paradise, M., Cochran, B. N., Shea, J. M., Srebnik, D., et al. (2002). Cultural and contextual influences in mental health help seeking: a focus on ethnic minority youth. J. Consult. Clin. Psychol. 70, 44-55. doi: 10.1037/0022-006x.70.1.44

Ceballos, M., Wallace, G., and Goodwin, G. (2016). Postpartum depression among African-American and Latina mothers living in small cities, towns, and rural communities. J. Rac. Ethnic Health Dispar. 4, 916-927. doi: 10.1007/s40615016-0295-Z

Chang, Y., and Fine, M. A. (2007). Modeling parenting stress trajectories among low-income young mothers across the child's second and third years: Factors accounting for stability and change. J. Fam. Psychol. 21, 584-594. doi: 10.1037/ 0893-3200.21.4.584

Chaput, J. P., and Janssen, I. (2016). Sleep duration estimates of Canadian children and adolescents. J. Sleep Res. 25, 541-548. doi: 10.1111/jsr.12410

Dennis, C. L., Coghlan, M., and Vigod, S. (2013). Can we identify mothers at-risk for postpartum anxiety in the immediate postpartum period using the StateTrait Anxiety Inventory? J. Affect. Disord. 150:49. doi: 10.1016/j.jad.2013.05. 049

Fagan, J., and Lee, Y. (2010). Perceptions and satisfaction with father involvement and adolescent mothers' postpartum depressive symptoms. J. Youth Adoles. 39, 1109-1121. doi: 10.1007/s10964-009-9444-6

Figueiredo, B., Pacheco, A., and Costa, R. (2007). Depression during pregnancy and the postpartum period in adolescent and adult Portuguese mothers. Arch. Womens Mental Health 10, 103-109. doi: 10.1007/s00737-007-0178-8

Goyal, D., Gay, C., and Lee, K. A. (2010). How much does low socioeconomic status increase the risk of prenatal and postpartum depressive symptoms in first-time mothers? Womens Health Issues 20, 96-104. doi: 10.1016/j.whi.2009.11.003 
Grace, S. L., Evindar, A., and Stewart, D. E. (2003). The effect of postpartum depression on child cognitive development and behavior: a review and critical analysis of the literature. Arch. Womens Mental Health 6, 263-274. doi: 10.1007/ s00737-003-0024-6

Hahn-Holbrook, J., Cornwell-Hinrichs, T., and Anaya, I. (2018). Economic and health predictors of national postpartum depression prevalence: a systematic review, meta-analysis and meta-regression of 291 studies from 56 countries. Front. Psychiatry 8:248. doi: 10.3389/fpsyt.2017.00248

Hirshkowitz, M., Whiton, K., Albert, S. M., Alessi, C., Bruni, O., DonCarlos, L., et al. (2015). National Sleep Foundation's sleep time duration recommendations: methodology and results summary. Sleep Health 1, 40-43. doi: 10.1016/j.sleh.2014.12.010

Howell, E. A., Mora, P. A., Horowitz, C. R., and Leventhal, H. (2005). Racial and ethnic differences in factors associated with early postpartum depressive symptoms. Obstetr. Gynecol. 105, 1442-1450. doi: 10.1097/01.aog.0000164050. 34126.37

Huang, C. Y., Roberts, Y. H., Costeines, J., and Kaufman, J. S. (2019). Longitudinal trajectories of parenting stress among ethnic minority adolescent mothers. J. Child Fam. Stud. 28, 1368-1378. doi: 10.1007/s10826-019-01356-1

Jahromi, L. B., Umaña-Taylor, A. J., Updegraff, K. A., and Lara, E. E. (2012). Birth characteristics and developmental outcomes of infants of Mexican-origin adolescent mothers: risk and promotive factors. Int. J. Behav. Dev. 36, 146-156. doi: 10.1177/0165025411430777

Kalak, N., Lemola, S., Brand, S., Holsboer-Trachsler, E., and Grob, A. (2014). Sleep duration and subjective psychological well-being in adolescence: a longitudinal study in Switzerland and Norway. Neuropsych. Dis. Treat. 10, 1199-1207. doi: 10.2147/NDT.S62533

Kaplan, C. P., Erickson, P. I., and Juarez-Reyes, M. (2002). Acculturation, gender role orientation, and reproductive risk-taking behavior among Latina adolescent family planning clients. J. Adoles. Res. 17, 103-121. doi: 10.1177/ 0743558402172001

Lanzi, R. G., Bert, S. C., and Jacobs, B. K. (2009). Depression among a sample of first time adolescent and adult mothers. J. Child Adoles. Psychiatry Nurs. 22, 194-202. doi: 10.1111/j.1744-6171.2009.00199.x

Luis Sanchez, B. E., Urbina, E., and D'Anna-Hernandez, K. L. (2020). Sociocultural stressors across the perinatal period and risk for postpartum depressive symptoms in women of Mexican descent. Cult. Diver. Ethnic Minor. Psychol. 26, 124-133. doi: 10.1037/cdp0000278

Oberlander, S. E., Black, M. M., and Starr, R. H. (2007). African American adolescent mothers and grandmothers: a multigenerational approach to parenting. Am. J. Commun. Psychol. 39, 37-46.

Office of Disease Prevention and Health Promotion (n.d.). Adolescent Health. Healthy People 2020. Washington, DC: U.S. Department of Health and Human Services.

Okun, M. L., Mancuso, R. A., Hobel, C. J., Schetter, C. D., and Coussons-Read, M. (2018). Poor sleep quality increases symptoms of depression and anxiety in postpartum women. J. Behav. Med. 41, 703-710. doi: 10.1007/s10865-0189950-7

Radloff, L. S. (1977). The CES-D scale: a self-report depression scale for research in the general population. Appl. Psychol. Measur. 1, 385-401. doi: 10.1177/ 014662167700100306

Raniti, M. B., Waloszek, J. M., Schwartz, O., Allen, N. B., and Trinder, J. (2018). Factor structure and psychometric properties of the Pittsburgh Sleep Quality Index in community-based adolescents. Sleep 41:zsy066. doi: 10.1093/sleep/ zsy066

Reichner, C. A. (2015). Insomnia and sleep deficiency in pregnancy. Obstet. Med. 8, 168-171. doi: 10.1177/1753495X15600572

Reid, V., and Meadows-Oliver, M. (2007). Postpartum depression in adolescent mothers: an integrative review of the literature. J. Pediatr. Health Care 21, 289-298. doi: 10.1016/j.pedhc.2006.05.010

Richter, D., Krämer, M. D., Tang, N. K., Montgomery-Downs, H. E., and Lemola, S. (2019). Long-term effects of pregnancy and childbirth on sleep satisfaction and duration of first-time and experienced mothers and fathers. Sleep 42:zsz015. doi: 10.1093/sleep/zsz015

Sadeghi Bahmani, D., Esmaeili, L., Shaygannejad, V., Gerber, M., Kesselring, J., Lang, U. E., et al. (2018). Stability of mental toughness, sleep disturbances, and physical activity in patients with Multiple Sclerosis (MS)-A longitudinal and pilot study. Front. Psychiatry 9:182. doi: 10.3389/fpsyt.2018.00182

Sasser, J., Lecarie, E. K., Park, H., and Doane, L. D. (2020). Daily family connection and objective sleep in Latinx adolescents: the moderating role of familism values and family communication. J. Youth Adoles. 20, 1-15. doi: 10.1007/s10964-02001326-7

Schmidt, R. M., Wiemann, C. M., Rickert, V. I., and Smith, E. O. (2006). Moderate to severe depression symptoms among adolescent mothers followed four years postpartum. J. Adoles. Health 38, 712-718. doi: 10.1016/j.jadohealth.2005.05. 023

Shochat, T., Cohen-Zion, M., and Tzischinsky, O. (2014). Functional consequences of inadequate sleep in adolescents: a systematic review. Sleep Med. Rev. 18, 75-87. doi: 10.1016/j.smrv.2013.03.005

Slomian, J., Honvo, G., Emonts, P., Reginster, J., and Bruyère, O. (2019). Consequences of maternal postpartum depression: a systematic review of maternal and infant outcomes. Womens Health 15, 1-55. doi: 10.1177/ 1745506519844044

Smith, E. N., Grau, J. M., Duran, P. A., and Castellanos, P. (2013). Maternal depressive symptoms and child behavior problems among Latina adolescent mothers: the buffering effect of mother-reported partner childcare involvement. Mer. Palmer Q. 59, 304-329. doi: 10.1353/mpq.2013.0014

Spielberger, C. D., Edwards, C. D., Lushene, R. E., Montuori, J., and Platzek, D. (1973). Preliminary Test Manual for the State-Trait Anxiety Inventory for Children. Palo Alto, CA: Consulting Psychologists Press.

Stiles, A. S. (2010). Case study of an intervention to enhance maternal sensitivity in adolescent mothers. J. Obstetr. Gynecol. Neonat. Nurs. 39, 723-733. doi: 10.1111/j.1552-6909.2010.01183.x

Stockings, E., Degenhardt, L., Lee, Y. Y., Mihalopoulos, C., Liu, A., Hobbs, M., et al. (2015). Symptom screening scales for detecting major depressive disorder in children and adolescents: a systematic review and meta-analysis of reliability, validity and diagnostic utility. J. Affect. Disord. 174, 447-463. doi: 10.1016/j.jad. 2014.11.061

Stremler, R., McMurray, J., and Brennenstuhl, S. (2019). Self-reported sleep quality and actigraphic measures of sleep in new mothers and the relationship to postpartum depressive symptoms. Behav. Sleep Med. 18, 396-405. doi: 10.1080/ 15402002.2019.1601629

Suarez-Colorado, Y. P., Caballero-Dominguez, C. C., and Campo-Arias, A. (2020). Confirmatory factor analysis and internal consistency of the suicidal ideation scale of the Center for Epidemiological Studies Depression among Colombian Aadolescents. Psychol. Rep. 3:33294120971771. doi: 10.1177/ 0033294120971771

Trejos-Herrera, A. M., Bahamón, M. J., Alarcón-Vásquez, Y., Vélez, J. I., and Vinaccia, S. (2018). Validity and reliability of the multidimensional scale of perceived social support in colombian adolescents. Psychos. Intervent. 27, 5663. doi: 10.5093/pi2018a1

Zhang, J., Paksarian, D., Lamers, F., Hickie, I. B., He, J., and Merikangas, K. R. (2017). Sleep patterns and mental health correlates in US adolescents. J. Pediatr. 182, 137-143. doi: 10.1016/j.jpeds.2016.11.007

Zhang, J., Xu, Z., Zhao, K., Chen, T., Ye, X., Shen, Z., et al. (2018). Sleep habits, sleep problems, sleep hygiene, and their associations with mental health problems among adolescents. J. Am. Psychiatr. Nurs. Assoc. 24, 223-234. doi: 10.1177/ 1078390317715315

Zimet, G. D., Dahlem, N. W., Zimet, S. G., and Farley, G. K. (1988). The multidimensional scale of perceived social support. J. Pers. Asses. 52, 30-41. doi: 10.1207/s15327752jpa5201_2

Conflict of Interest: The authors declare that the research was conducted in the absence of any commercial or financial relationships that could be construed as a potential conflict of interest.

Copyright (c) 2021 Yung, Main, Walle, Scott and Chen. This is an open-access article distributed under the terms of the Creative Commons Attribution License (CC BY). The use, distribution or reproduction in other forums is permitted, provided the original author(s) and the copyright owner(s) are credited and that the original publication in this journal is cited, in accordance with accepted academic practice. No use, distribution or reproduction is permitted which does not comply with these terms. 